\title{
La pitanga y el mangusán, tesoros gastronómicos de Colombia
}

\section{The pitanga and the mangusán, gastronomic treasures from Colombia}

\author{
Diana Margoht González Ramírez* \\ Fundación Universitaria San Mateo \\ Bogotá, Colombia
}

Angie Lorena Hernández Bolaños**

Fundación Universitaria San Mateo

Bogotá, Colombia

\author{
Alba Lucia Muñoz Mesa*** \\ Fundación Universitaria San Mateo \\ Bogotá, Colombia \\ Javier Eduardo Sánchez Ramos**** \\ Fundación Universitaria San Mateo \\ Bogotá, Colombia
}

Luis Ignacio Martínez ( $†$ )

Fundación Universitaria San Mateo

Bogotá, Colombia

*dmargothgonzalez@sanmateo.edu.co

**alhernandez@sanmateo.edu.co

***almunozm@sanmateo.edu.co

****jesanchez@sanmateo.edu.co

\section{Articulo de investigación}

Recepción: 30 de julio de 2019. Aceptación: 21 de agosto de 2019

\section{Cómo citar este artículo}

Gonzáles, D; et al. (2019). La Pitanga y el Mangusán, tesoros gastronómicos de Colombia. Sosquua 1(1), 14-21. Recuperado a partir de http://cipres.sanmateo.edu.co/index.php/sosquua 


\section{Resumen}

En el desarrollo de esta investigación se busca analizar las potencialidades gastronómicas que tiene la Pitanga y el Magusan dentro de las ofertas gastronómicas nacionales. Como punto de partida se llegó a las poblaciones de la Mesa y Cáqueza. En el presente artículo se describen las principales características organolépticas, además de identificar preparaciones que ayudan a difundir la riqueza de estos productos dentro de la generación de una identidad culinaria local y nacional. A través de una recopilación de elementos pedagógicos, se establecen las características de formación que deben cumplir los profesionales de cocina dentro del reconocimiento cultural y geográfico de las regiones del país.

Palabras clave: gastronomía, pitanga, magusán, identidad gastronómica.

\section{Abstract}

In the development of this research, we sought to analyze the gastronomic potentials that Pitanga and Magusan have within the national gastronomic offerings. As starting point, we got around the populations of Mesa and Cáqueza. The main organoleptic characteristics were described, in addition to identifying preparations that help spread the richness of these products within the generation of a local and national culinary identity. Through a compilation of pedagogical elements, the training characteristics that cooking professionals must fulfill within the cultural and geographical recognition of the regions of the country were established.

Keywords: gastronomy, pitanga, magusán, gastronomic identity. 


\section{Introducción}

Dentro de la búsqueda de establecer el reconocimiento de una identidad gastronómica en los cocineros profesionales, y de generar la salvaguarda de tradiciones, se toma la iniciativa de explorar la importancia de analizar los frutos de la Pitanga y del Magusan en las poblaciones de la Mesa y Cáqueza en el departamento de Cundinamarca, Colombia. Lo anterior, por medio del enaltecimiento de las características de los productos en la generación de una nueva oferta gastronómica que permita fortalecer las poblaciones dentro del mapa culinario nacional.

Para ello, se describen las características de la pitanga como fruta tropical que se presenta en los bosques de Brasil. Sin embargo, a través de la transculturización ocurrida por los alemanes en los años 60, dicha fruta ingresa al municipio de la Mesa para su cultivo como elemento decorativo de los jardines de las casas de los pobladores (Babiloni, 2012). La palabra Pitanga significa "fruta roja de Surinam cherry". Su cosecha se da con mayor frecuencia entre el verano y el otoño. Es un producto con un alto desarrollo de elementos aromáticos y con una semilla grande que permiten su fácil propagación (Instituto Naconal de Investigación Agropecuaria, 2012).

El magusán, por su parte, se conoce comúnmente como "zapote blanco". Es una especie arbórea perteneciente a la familia Rutaceae, originaria de México, adventicia y naturalizada en los valles interandinos y occidentales del Perú (Frazon, 2008). El Magusan llegó al país mediante el intercambio de especias que se establecieron en la colonización española.

Estas frutas son poco conocidas dentro del sector de la cocina nacional, ya que la mayoría de las ocasiones se utilizaban como elementos decorativos en festividades o días culturales de los municipios. Mediante un proceso etnográfico, se tomó la iniciativa de acudir a los destinos de producción con el fin de conocer, de primera mano, su cultivo y utilización dentro de los contextos generados en la tradición de la población nativa. 


\section{Resultados}

Dentro del desarrollo de la indagación de los productos del Magusán, se acudió al Instituto de Recurso Biológicos Alexander Von Humboldt. Con la descripción de la fruta, se buscó identificar sus principales características de cultivo. Sin embargo, el instituto no poseía información de fiabilidad y desconocían su procedencia dentro del territorio nacional.

A partir de lo expuesto por el Instituto Alexander Von Humboldt, se inició con el estudio bromatológico de la fruta, realizado por el laboratorio NULAB Ltda., quien determinó la siguiente información:

Figura 1. Resultado estudio de bromatología.

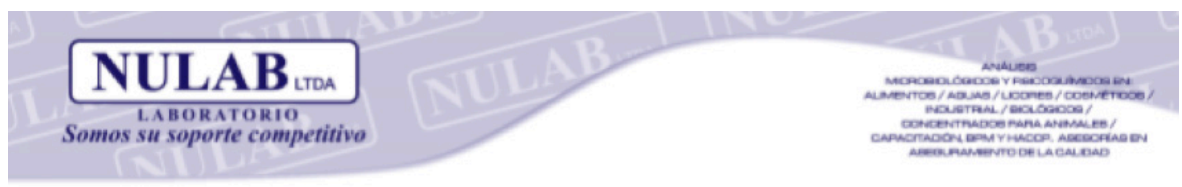

Reporte de Análisis Fisicoquímico 20181010680

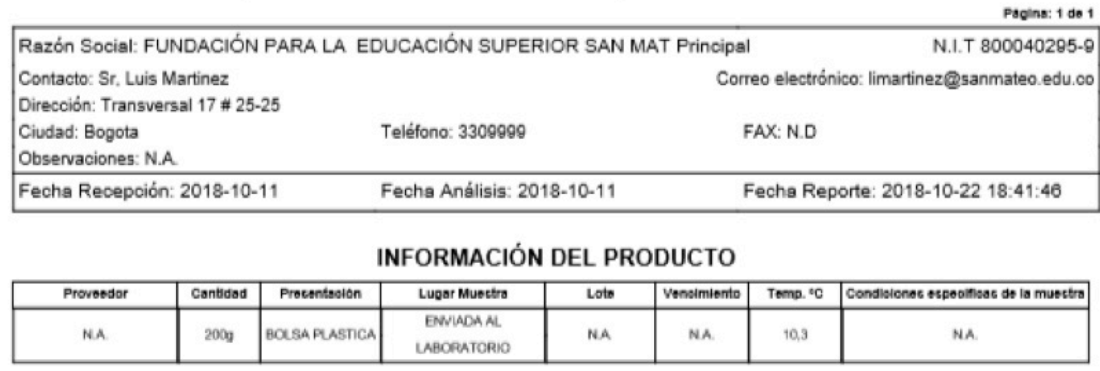

\begin{tabular}{|c|c|c|c|}
\hline \multicolumn{4}{|c|}{ MAGUZAN (FRUTA) (A0680) } \\
\hline ANALU:18 & REBULTADOB & PARAMETRO & METODO ANALIEIS \\
\hline CALORIAS & $78.37 \mathrm{kcad} / 00 \mathrm{~g}$ & Nobspicas & NTC 512.2 \\
\hline CARBOHIDRATOS & $17.76 \mathrm{~g} 100 \mathrm{~g}$ & No aplica & NTC 512:2 \\
\hline CEMIZAS & $0.00 \mathrm{~g} 100 \mathrm{~g}$ & No aptica & Calcinacion \\
\hline FIBRA CALOA & 1.50 g100g & No applica & AOAC 96209 \\
\hline GPASA TOTAL & $0.069100 \mathrm{~g}$ & No aplica & NTC G6S \\
\hline HUMEDAD & $78.599100 \mathrm{~g}$ & Nob aploa & Secado en estifia \\
\hline PROIEIMÁ & $1.22 \mathrm{~g} 100 \mathrm{~g}$ & No aptica & Keidairi \\
\hline SOLIDOS TOTALES & $21.419100 \mathrm{~g}$ & No aptea & 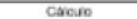 \\
\hline
\end{tabular}

Informadion fsicoquimica en $100 \mathrm{~g}$ de munsta andizada.

FIN DEL REPORTE

Fuente: Laboratorio NULAB, 2018.

\footnotetext{
${ }^{1}$ Estudio bromatológico que establece la importancia de las características de clasificación de los frutaslas frutas a ni Colombia
} 
Luego de los resultados expuestos por el estudio, se evidenció que la fruta no es nativa de la localidad de Caqueza (Rodríguez-Parra y Acosta-Triviño, 2017). El fruto de esta región es la unión de injertos entre varias frutas que, mediante la evolución del territorio, solamente ha sido reconocido de forma decorativa dentro de la población.

En el caso de la pitanga se encontró que, mediante procesos químicos, las propiedades de esta fruta sirven para el desarrollo de productos cosméticos. Gracias a su gran abundancia de aceite de optima pureza, garantiza la calidad y densidad del material para la belleza.

\section{Discusión de resultados}

Según el interés por el desarrollo de preparaciones gastronómicas con la utilización de estos dos frutos, y tomando como referencia los hallazgos encontrados, se puede determinar que dentro de las preparaciones de la futa del magusán se puede realizar jugos por medio de la industrialización de la pulpa en conservas. Con frecuencia, también se consume de manera directa. La pulpa de los frutos maduros se puede agregar a cocteles de frutas y ensaladas o como postre servido solo. Sin embargo, lo mejor es cortado en secciones y servido con crema y azúcar.

(...) A veces se añade a las mezclas para helados o batidos con leche, o se hace en mermelada. Incluso en sus países de origen, donde los frutos pueden a veces aparecer en los mercados, su reputación se debe en gran parte a la creencia en su valor terapéutico, mientras que, al mismo tiempo, prevalece el temor de que los excesos pueden ser perjudiciales. (Filho, 2018, p. 36) 
En el caso de la fruta pitanga su uso se ve reflejado en preparaciones no solo para la belleza, siendo un fruto rico en vitamina A, calcio, fósforo y hierro; también se evidencia en preparaciones comestibles elaboradas por indígenas a partir de las hojas, en pequeñas cantidades, logrando infusiones benéficas para la salud (Krauss, 2018). "En Argentina se produce caña de ñangapirí, infusionando los frutos con aguardiente. Con sus zumos también puede producirse una especie de vino o vinagre. La fruta puede comerse fresca, prepararse en dulce o jalea" (INNATIA, 2018).

Del mismo modo, el área de la medicina utiliza este árbol para infusiones diuréticas y digestivas que ayudan al organismo (Dime Beneficios, 2018). En gastronomía es empleada para comerse fresca hasta hacer conservas, jaleas, mermeladas y jugos. Además, como se mencionaba anteriormente, en Uruguay y Argentina se emplea para hacer una bebida alcohólica a base del jugo de la pitanga que, si se deja fermentar, puede llegar a ser un vino o vinagre de esta misma (Todo Uruguay, 2018; INNATIA, 2018).

Actualmente, la industria de dulces se encuentra realizando pruebas experimentales con la pitanga. Por su parte, en la industria láctea, la pulpa de pitanga es apta para yogures, helados, jugos y licuados. En este caso, se trabaja con pulpa a $42^{\circ} \mathrm{Br}{ }^{2} i x$ y se resaltó la textura, el color y el sabor. Los frutos se manejaron congelados luego de su cosecha. En el caso de la industria láctea o de las bebidas, es necesario suministrar la materia prima ya elaborada, por lo que debe necesariamente tener un proceso industrial previo para obtener la pulpa, pasta, mermelada o jugo requerido para su aprovechamiento, lo implica disponer de instalaciones habilitadas para dicho propósito (Vignale, Jolochin \& Cabrera, 2018).

\footnotetext{
242 grados Brix punto de inicio para la elaboración de bebidas.
} 


\section{Conclusiones}

Como el cultivo del Magusán (Zapote Blanco) es de carácter estacional. Se recomienda validar con anticipación sus meses de cosecha, para poder realizar las recetas sin tener inconvenientes en la generación de nuevos menús.

Dado que son frutos poco conocidos a nivel local y nacional, se recomienda explorar mucho más sobre esta fruta a nivel investigativo y crear preparaciones gastronómicas que hagan más reconocida esta fruta. Implica, por ende, incentivar su cultivo, comercialización y consumo.

A nivel de gastronomía, es importante indagar los principales ingredientes de las regiones de Colombia. A partir de esa caracterización, generar nuevas ofertas gastronómicas que ayuden al reconocimiento de una identidad gastronómica colombiana.

\section{Lista de referencias}

Babiloni, J. (28 de 01 de 2012). Jardin Mundani. Recuperado de: http://jardin-mundani.blogspot.com/2012/01/eugenia-uniflora-pitanga-del-surinam.html

DIME BENEFICIOS. (18 de 09 de 2018). Dime Beneficios propiedades de la pitanga. Recuperado de https://www.dimebeneficios. com/propiedades-de-la-pitanga/

INNATIA. (15 de septiembre de 2018). INNATIA. Recuperado de: http://www.innatia.com/s/c-frutas-propiedades-frutos/a-propiedades-del-nangapiri.html

Filho, J. (2018). Zapote blanco. Sabelotodo.org. Recuperado de: http://www.sabelotodo.org/agricultura/frutales/sapoteblanco. html 
Instituto Nacional de Investigación Agropecuaria. (2012). "Ficha Temática Número 2. Nuestros Frutos Nativos. Pitanga." Recuperado de: http://www.inia.org.uy/online/site/web/articulos/ Ficha\%202\%20Pitanga.pdf

Krauss, L. R. (17 de abril de 2017). ¿Conocías los aportes nutricionales del fruto de la pitanga? Misiones Online. Recuperado de http://misionesonline.net/2017/04/23/conocias-los-aportes-nutricionales-del-fruto-la-pitanga/

Rodríguez-Parra, P. y Acosta-Triviño, R. (2017). Delitos y conflicto en la localidad 16 Puente Aranda. Bogotá: San Mateo Educación Superior. Recuperado de https://palma.sanmateo.edu.co/

Todo Uruguay. (15 de septiembre de 2018). Hojas de pitanga, el espantamoscas natural. Todo Uruguay. Recuperado de: https://www.todouruguay.net/hojas-de-pitanga-el-espantamoscas-natural/

Vignale, B., Jolochin, G., \& Cabrera, D. (2018). Eugenia uniflora L. Pitanga, Ñangapiré, Pitangueira, Pitanguero, Surinam cherry, Cayenne cherry. PITANGA. 4-21. 